Prepared For SUbMission to JCAP

\title{
Reacceleration of charged dark matter
}

\author{
M. Kachelrieß and J. Tjemsland \\ Institutt for fysikk, NTNU, Trondheim, Norway
}

Received: June 15, 2020

Accepted: July 31, 2020

\begin{abstract}
Charged particles scattering on moving inhomogenities of the magnetised interstellar medium can gain energy through the process of second-order Fermi acceleration. This energy gain depletes in turn the magnetic wave spectrum around the resonance wave-vector $k \sim 1 / R_{L}$, where $R_{L}$ is the Larmor radius of the charged particle. This energy transfer can prohibit the cascading of magnetic turbulence to smaller scales, leading to a drop in the diffusion coefficient and allowing the efficient exchange of charged dark matter particles in the disk and the halo. As a result, terrestial limits from direct detection experiments apply to charged dark matter. Together with the no-observation of a drop in the diffusion coefficient, this excludes charged dark matter for $10^{3} \mathrm{GeV} \lesssim m / q \lesssim 10^{11} \mathrm{GeV}$, even if the charged dark matter abundance is only a small part of the total relic abundance.
\end{abstract}

Keywords: dark matter, reacceleration, cosmic ray, diffusion, Alfvén waves 


\section{Contents}

1 Introduction 1

2 Alfvén waves and diffusion 2

2.1 Diffusion equations 2

2.2 Resonance condition 3

$\begin{array}{ll}2.3 & \text { Diffusion coefficients }\end{array}$

3 Power taken by millicharged dark matter 4

4 Key rates $\quad \mathbf{5}$

$\begin{array}{lll}4.1 & \text { Growth rate } & 5\end{array}$

$\begin{array}{lll}4.2 & \text { Damping, diffusion and advection rates } & 6\end{array}$

$\begin{array}{lll}4.3 & \text { Thermalisation rate } & 6\end{array}$

$\begin{array}{lll}4.4 & \text { Supernova shock encounter rate } & 6\end{array}$

$\begin{array}{lll}4.5 & \text { Injection rate of turbulence } & 6\end{array}$

5 Time evolution and observable consequences $\quad 7$

$\begin{array}{lll}5.1 & \text { Time evolution } & 7\end{array}$

$\begin{array}{lll}5.2 & \text { Observable consequences } & 7\end{array}$

6 Conclusions $\quad 9$

\section{Introduction}

There is overwhelming evidence for the presence of a non-baryonic component in the matter budget of the Universe through its gravitational effects. If this component consists of particles having only gravitational interactions, there would be little hope for their detection. Therefore it is commonly assumed that they participate in part of the gauge interactions of the standard model (SM). In order to keep the particles dark and collisionless, typically the weak interaction is chosen as coupling to the SM particles. However, no new weakly interacting particles have been detected yet, despite of intense searches. As a consequence, the interest in alternatives has been growing. One option is the case of millicharged dark matter (DM), where the DM particle has a small, but non-zero electric charge $q e \ll e$, thereby providing a coupling via the photon to the SM particles [1]. The smallness of $q$ could be explained, e.g., by the kinetic mixing of the dark photon of an extra U(1) symmetry with the SM photon [2-6]. Alternatively, DM with charges of order one could form heavy bound states [7].

The case of millicharged DM has obtained increased attention lately following the reported discrepancy between predictions for the $21 \mathrm{~cm}$ absorption line induced by early stars and observations by the EDGES collaboration [8]. It has been suggested that this anomaly is caused by the cooling of baryons at redshift $z \simeq 17$ in baryon-dark matter interactions with a massless mediator [9]. Dark matter with a tiny electric charge $q$ would be a natural candidate for such a new cooling agent. Unfortunately, a scenario where the EDGES observations are explained by millicharged DM that explains all the observed DM abundance is inconsistent with bounds from the cosmic microwave background [10]. Therefore, DM theories in which there is a sub-dominant charged component, such as theories including a completely hidden sector have attracted attention, see e.g. Refs. [11-14] for recent examples and Refs. [15, 16] for reviews.

Cosmological observations and direct detection experiments put strong constraints on the possible mass-to-charge ratios of DM [17, 18]. However, Chuzhoy and Kolb [19] claimed in 2009 that the direct detection constraints on charged DM are invalid in a wide range of $\mathrm{m} / \mathrm{q}$ values: They argued 
that the regular ${ }^{1}$ Galactic magnetic field (GMF) in the disk prevents charged DM particles with $m / q \lesssim 10^{11} \mathrm{GeV}$ from entering the plane from the halo. Taking in turn into account the acceleration of charged DM particles in the disk by shock fronts of supernova remnants and the subsequent loss of energetic DM particles into the halo, they argued that charged DM with $10^{5} q^{2} \lesssim m / G e V \lesssim 10^{11} q$ is expelled from the disk and evades thereby the bounds from terrestrial direct DM searches. This range was later reduced to $m / q \lesssim 10^{9} \mathrm{GeV}$ in Ref. [20] by taking into account the effect of the turbulent magnetic field and the non-homogeneity of the background field. The authors of Ref. [21] argued that the injection of charged DM into diffusive shock acceleration is suppressed, implying that these particles are not effectively accelerated in shock fronts of supernova remnants. The most recent and complete study treating charged DM as diffusive cosmic rays has been preformed in Ref. [22]. In this analysis, it was found that there is a substantial amount of charged DM present in the Galactic disk today, some of which have recently been accelerated. This was used to set strong constraints on the possible $(m, q)$ parameters.

In this work, we consider a generic DM particle with mass $m$ and charge qe. As any electrically charged particle, charged DM will scatter on the inhomogeneities of the turbulent GMF. These inhomogeneities are moving with typical velocities of tens of $\mathrm{km} / \mathrm{s}$ with respect to the Galaxy, either because static turbulence is advected alongside the plasma or because the turbulence consists of travelling Alfvén waves. Both cases lead to second-order Fermi acceleration of charged particles including charged DM. The energy gained by the charged DM depletes in turn the magnetic wave spectrum around the resonance wave-vector $k_{\text {res }} \sim 1 / R_{L}$, where $R_{L}$ is the Larmor radius of the charged DM particle. If this energy drain is larger than the power injected into the turbulent GMF at the injection wave-number $k_{\min }$, the cascading of magnetic turbulence from $k_{\min }$ to larger wave-numbers is stopped at $k_{\text {res }}$. In this case, all fluctuations in the inertial range above $k_{\text {res }}$ are missing. As a result, charged DM from the halo can enter the disk and the limits from direct DM searches can be applied. Together with the no-observation of a drop in the diffusion coefficient, this excludes charged DM for a wide range of masses and charges.

\section{Alfvén waves and diffusion}

\subsection{Diffusion equations}

The propagation of charged particles through the magnetized interstellar medium (ISM) filling the Milky Way can be described phenomenologically as a combination of diffusion and advection using a Fokker-Planck equation [23, 24]: The scattering of charged particles on the inhomogeneities of the turbulent GMF leads to diffusion terms in the evolution equation for the phase space density $f(\boldsymbol{x}, \boldsymbol{p})$ of the charged DM particle,

$$
\frac{\partial f}{\partial t}=Q+\nabla(D(p) \nabla f)+\frac{1}{4 \pi p^{2}} \frac{\partial}{\partial p}\left(4 \pi p^{2} D_{p p}(p) \frac{\partial f}{\partial p}\right)+\ldots
$$

Here, $D$ and $D_{p p}$ parametrise diffusion in position and momentum space, respectively, while $Q$ is a source term and $p=|\boldsymbol{p}|$ the momentum of the charged DM particle. Moreover, we assumed for simplicity that the diffusion is isotropic, i.e. we replaced the diffusion tensor $D_{i j}(\boldsymbol{p})$ by the scalar diffusion coefficient $D(p)$. Additionally, charged particles are advected with the plasma.

Alfvén waves are solutions of the MHD equations which propagate approximately parallel to the magnetic field lines with the Alfvén velocity ${ }^{2} v_{A}=B_{0} / \sqrt{4 \pi \rho} \simeq 30 \mathrm{~km} / \mathrm{s}$, where $\rho$ is the density of the plasma and $B_{0}$ the strength of the magnetic background field. Charged particles are dynamically coupled to the ISM via the self-generation of, and the scattering on, Alfvén waves. Thus, the usual "test-particle" approach, where charged particles propagate in a prescribed static background (or given diffusion coefficients $D$ and $D_{p p}$ in the diffusion picture) is in general not valid. Instead, one must check if their back-reaction on the turbulent magnetic field at scales comparable to their Larmor radius is negligible.

\footnotetext{
${ }^{1}$ Note that Liouville's theorem implies that the intensity of charged DM is constant along a trajectory in the magnetic field. Thus a shielding effect analogue to the geomagnetic cutoff would require, e.g., a dipole component of the GMF.

${ }^{2}$ We use Gaussian units and set $c=1$.
} 
The rate at which energy is transferred between Alfvén waves and charged particles is given by ${ }^{3}$

$$
\Gamma_{\text {growth }}=\frac{16 \pi^{2}}{3} \frac{p^{4} v_{A}}{k W_{k}(t) B_{0}^{2}}\left(v \hat{\boldsymbol{n}} \cdot \nabla f-\frac{\pi}{2} m \Omega_{0} v_{A} k W_{k}(t) \frac{\partial f}{\partial p}\right),
$$

where $\Omega=q e B_{0} / m$ is the cyclotron frequency of a particle with mass $m$ and charge $q e, v$ its velocity, and $W_{k}$ denotes the spectral density of turbulent field modes with wave-vector $\boldsymbol{k}$ [25]. This interaction proceeds resonantly, such that $k$ equals $k_{\text {res }} \simeq \Omega / v \mu$ with $\mu=\cos \vartheta$ as the cosine of the pitch angle [26]. We normalise the spectral density of turbulent field modes $W_{k}$ such that $\eta_{B} \equiv \delta B^{2} / B_{0}^{2}=\int \mathrm{d} k W(k)$, where $\eta_{B}$ denotes the ratio of the energy density in the turbulent and the regular magnetic field. Sources of this turbulence are the mechanical "stirring" of the plasma and the generation of Alfvén waves by charged particles. In the former case, stellar winds, supernova shocks and the differential rotation of the Milky Way inject energy into the ISM on scales of tens of parsecs, which then cascades down to smaller scales through the formation of smaller and smaller eddies. This energy cascade in the inertial range can be modelled as a diffusion process in $k$ space [27],

$$
\frac{\partial W_{k}(t)}{\partial t}=\frac{\partial}{\partial k}\left(D_{k k} \frac{\partial W_{k}(t)}{\partial k}\right)-\Gamma_{\text {growth }}(k, t) W_{k}(t)+q_{W},
$$

with the diffusion coefficient [28]

$$
D_{k k}=C_{K} v_{A} k^{\alpha_{1}} W_{k}(t)^{\alpha_{2}}
$$

and $C_{K} \simeq 0.052$ [29]. The injection occurs via the source term $q_{W} \propto \delta\left(k-2 \pi / L_{\max }\right)$ at the outer scale $L_{\max } \simeq 100$ pc. The parameters $\alpha_{i}$ are chosen as $\alpha_{1}=7 / 2$ and $\alpha_{2}=1 / 2$ such that the power-law spectrum

$$
W(k)=W_{0}\left(\frac{k}{k_{\min }}\right)^{-s}, \quad s=\frac{\alpha_{1}-1}{\alpha_{2}+1}, \quad W_{0}=(s-1) L_{\max } \eta_{B},
$$

obtained as steady-state solution for $\Gamma_{\mathrm{ex}}=0$ is a Kolmogorov spectrum. The wave-number $k_{\min }=$ $2 \pi / L_{\max }$ is determined by the injection scale $L_{\max }$ of the turbulence.

Equations (2.1) and (2.3) form a set of coupled differential equations that must be solved iteratively in order to compute the time evolution of both the phase-space density $f$ of charged DM and the spectrum $W_{k}$ of magnetic field fluctuations.

\subsection{Resonance condition}

Since the interaction (2.2) between charged particles and Alfvén waves proceeds resonantly, we have to check that the resonant wave-vector $k_{\text {res }}$ of the charged DM particle is contained in the inertial range $\left[k_{\min }: k_{\max }\right]$ of the turbulent cascade. Here, $k_{\max }=2 \pi / L_{\min }$ is given by the dissipation scale where the turbulent energy is converted into heat. While observations show that fluctuations extend down at least to $10^{9} \mathrm{~cm}[30,31]$, it is theoretically expected that the dissipation scale corresponds to the proton or even electron Larmor radius $[32,33]$. For concreteness, we will use in the following the proton Larmor radius as the dissipation scale.

The resonant wave-number for the momentum $p=\gamma m v$ is [26]

$$
k_{\mathrm{res}}=\frac{\Omega m}{p} \frac{1}{\mu \pm v_{A} / v} \geq \frac{\Omega m}{p} \frac{1}{1 \pm v_{A} / v} .
$$

We first check that the condition $k_{\text {res }}<k_{\max }$ is satisfied. Using that the maximum wave-number is limited by the proton cyclotron frequency, $k_{\max } \simeq \Omega_{\mathrm{p}} / v_{A}$, the momentum that can resonate with Alfvén waves must satisfy

$$
p \gtrsim m v_{A}\left(\frac{q m_{\mathrm{p}}}{m} \mp 1\right)
$$

in the non-relativistic case, $p=\gamma m v \approx m v$. In the for us interesting limit $m / q \gg m_{\mathrm{p}}$, this reduces ${ }^{4}$ to $p \gtrsim m v_{A}$. Since the virial velocity of the DM, $v_{\text {vir }} \sim 300 \mathrm{~km} / \mathrm{s}$, is much larger than the Alfvén velocity, the majority of the charged DM particles fulfills this condition.

\footnotetext{
${ }^{3}$ We assume that the forward and backward scattering rates are the same.

${ }^{4}$ The choice of sign means that the charged DM can resonate only with one of the two polarisation states of the Alfvén waves.
} 
Next we examine when the condition $k_{\text {res }}>k_{\min }$ is satisfied. Setting for simplicity $\mu=1$, it is $k_{\text {res }}=\Omega / v=1 / R_{L}$ with $R_{L}=\gamma m v / q e B_{0}$ as the Larmor radius. Thus, requiring

$$
R_{L} \simeq 1.08 \times 10^{-3} \mathrm{pc}\left(\frac{v_{\mathrm{vir}}}{300 \mathrm{~km} / \mathrm{s}}\right)\left(\frac{B_{0}}{1 \mu \mathrm{G}}\right)^{-1}\left(\frac{m / q}{10^{6} \mathrm{GeV}}\right) \lesssim L_{\max }
$$

with $L_{\max }=100 \mathrm{pc}$ gives the constraint

$$
m / q \lesssim 10^{11} \mathrm{GeV}
$$

\subsection{Diffusion coefficients}

The diffusion coefficients can be written as

$$
D=\frac{1}{3} \frac{v^{2}}{\nu_{+}+\nu_{-}} \quad \text { and } \quad D_{p p}=\frac{4}{3} \gamma^{2} m^{2} v^{2} v_{A}^{2} \frac{\nu_{+} \nu_{-}}{\nu_{+}+\nu_{-}}
$$

where $\nu_{ \pm}=\Omega k W(k) / \gamma$ are the collision frequencies of the forward and backward propagating Alfvén waves at the resonance $k_{\text {res }}$, and $\gamma$ is the Lorentz facor of the charged particle [25]. Assuming again that the forward and backwards rates are equal and inserting the expression (2.5) for the wave spectrum, the diffusion coefficients can be rewritten as

$$
D=\frac{v L_{\max }}{3}\left[\eta_{B}(s-1)\right]^{-1}\left(\frac{R_{L}}{L_{\max }}\right)^{2-s} \quad \text { and } \quad D_{p p}=\frac{1}{3} \gamma^{2} m^{2} v_{A}^{2} L_{\max } \eta_{B}(s-1)\left(\frac{R_{L}}{L_{\max }}\right)^{s-2},
$$

where $s=\left(\alpha_{1}-1\right) /\left(\alpha_{2}+1\right)=5 / 3$. Choosing then $\eta_{B} L_{\max } \simeq 1 \mathrm{pc}$, this framework is consistent with the commonly used parametrisation $D=D_{0} v(p / m)^{\delta}$ with $\delta=2-s$ and

$$
D_{0}=3 \times 10^{28} \frac{\mathrm{cm}^{2}}{\mathrm{~s}}\left[\left(\frac{m / q}{10^{6} \mathrm{GeV}}\right)\left(\frac{B}{1 \mu \mathrm{G}}\right)^{-1}\right]^{2-s} .
$$

\section{Power taken by millicharged dark matter}

The power density required for the reacceleration of cosmic rays is discussed in Ref. [34]. There, it is found that $\simeq 10 \%$ of the power in the cosmic ray proton spectrum comes from reacceleration and affects mainly the non- and mildly relativistic part of the cosmic ray spectrum. The same process must occur for charged DM, which means that its effect is potentially important. Following the procedure in Ref. [34] and using the diffusion coefficients given in section 2.3, we obtain as the reacceleration power density

$$
P_{R} \approx \frac{1}{9}(4-\delta) \frac{v_{A}^{2}}{D_{0}} m \int_{0}^{\infty} \mathrm{d} p 4 \pi p^{2}\left(\frac{p}{m}\right)^{1-\delta} f(p),
$$

where $f(p)$ is the momentum distribution of the particles. For comparison, we will consider protons with $f(p) \propto(p / m)^{-\gamma}$,

$$
P_{R}^{\text {protons }} \simeq 0.56 \mathrm{eV} / \mathrm{cm}^{3} \frac{v_{A}^{2}}{D_{0, \text { proton }}} .
$$

We consider a standard Maxwellian phase space density for the momentum distribution of the charged DM particles,

$$
f(p, z)=\left(2 \pi \varepsilon^{2}\right)^{-3 / 2} \exp \left\{-\frac{p^{2}}{2 \varepsilon^{2}}\right\} n(z)
$$

with $\varepsilon=\gamma m v_{\text {vir }} / \sqrt{2}$ and $n(z)=n_{0} \simeq 0.3(\mathrm{GeV} / m) / \mathrm{cm}^{3}$ as the local charged DM density. Thus, we obtain

$$
\frac{P_{R}}{P_{R}^{\text {protons }}}=4 \times 10^{6}\left(\frac{m}{10^{6} \mathrm{GeV}}\right)^{2 / 3} q^{1 / 3}\left(\frac{v \gamma}{300 \mathrm{~km} / \mathrm{s}}\right)^{1 / 2}\left(\frac{B}{1 \mu \mathrm{G}}\right)^{1 / 3} .
$$

This means that the power density going into the reacceleration of charged DM is potentially large, exceeding formally the available power density injected into the turbulent ISM. 


\section{Key rates}

From the estimate in the previous subsection, it is clear that the reacceleration of charged DM has the potential of seriously disturbing the ISM. In order to understand the potential consequences, we have to estimate the relevant time-scales of the problem, which will be discussed in this section. We will focus on the case where the entire relic density consists of charged DM, but we will comment on the case of a subdominant component in the next section. A summary of the results is shown in Fig. 1.

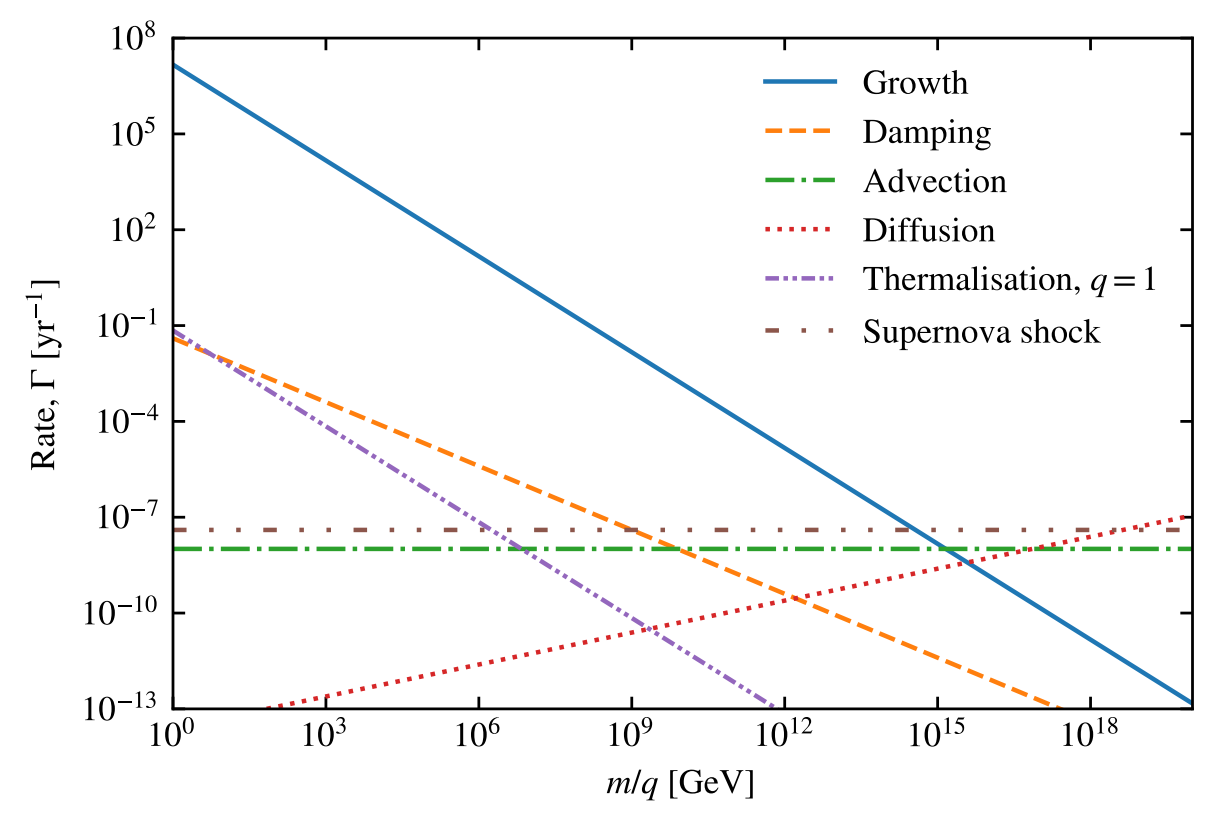

Figure 1. Various rates as a function of the charge-to-mass ratio of the charged DM.

\subsection{Growth rate}

In order to estimate the growth rate given in Eq. (2.2), we consider a Maxwellian phase space distribution (3.3). That is, the momentum distribution becomes $4 \pi p^{2} f(p, z)$ so that the number of particles in the momentum range $(p, p+\mathrm{d} p)$ is $4 \pi p^{2} f(p, z)$.

With $\partial_{z} f \sim\left[n_{0} / H\right] \cdot[f / n(z)]$ and choosing $H \sim 3 \mathrm{kpc}$ as the half-height of the confinement region, the growth rate induced by the spatial gradient is

$$
\begin{aligned}
\Gamma_{\text {growth }}^{z} & =\frac{2.4}{\mathrm{yr}}\left(\frac{m / q}{10^{6} \mathrm{GeV}}\right)^{-2 / 3}\left(\frac{v}{300 \mathrm{~km} / \mathrm{s}}\right)^{4 / 3} \gamma^{1 / 3} \exp \left\{-\frac{p^{2}}{p_{\text {vir }}^{2}}\right\} \\
& \left(\frac{B_{0}}{1 \mu \mathrm{G}}\right)^{-4 / 3}\left(\frac{v_{A}}{30 \mathrm{~km} / \mathrm{s}}\right)\left(\frac{\eta_{B}}{0.05}\right)^{-1}\left(\frac{L_{\mathrm{max}}}{50 \mathrm{pc}}\right)^{2 / 3}\left(\frac{H}{3 \mathrm{kpc}}\right)^{-1} .
\end{aligned}
$$

Likewise, using $n(z) \sim n_{0}$, the momentum gradient leads to the growth rate

$$
\Gamma_{\text {growth }}^{p}=\frac{40}{\mathrm{yr}}\left(\frac{m / q}{10^{6} \mathrm{GeV}}\right)^{-1} \frac{p}{p_{\text {vir }}} \exp \left\{-\frac{p^{2}}{p_{\text {vir }}^{2}}\right\}\left(\frac{B_{0}}{1 \mu \mathrm{G}}\right)^{-4 / 3}\left(\frac{v_{A}}{30 \mathrm{~km} / \mathrm{s}}\right)^{2} .
$$

Since we in our picture start without any spatial gradient and the corresponding growth rate $\Gamma_{\text {growth }}^{z}$ is small even assuming a large gradient, it can be neglected relative to $\Gamma_{\text {growth }}^{p}$. It is apparent from Fig. 1 that the growth rate is dominant for $m / q \lesssim 10^{15} \mathrm{GeV}$, where it has been evaluated using $p=p_{\text {vir }}$ and the numerical values of the astrophysical parameters given in Eqs. (4.1) and (4.2). 


\subsection{Damping, diffusion and advection rates}

The relevant rates in the diffusion equations describing the propagation of charged DM particles and their interaction with the wave spectrum are the damping rate $\Gamma_{\text {damp }} \sim D_{k k} / k^{2}$, the advection rate $\Gamma_{\mathrm{adv}} \sim v_{\mathrm{adv}} / H$ and the diffusion rate $\Gamma_{\mathrm{diff}} \sim 2 D / H^{2}$. Numerically, we can estimate these rates as

$$
\begin{aligned}
\Gamma_{\text {damp }}= & \frac{4 \times 10^{-6}}{\mathrm{yr}}\left(\frac{m / q}{10^{6} \mathrm{GeV}}\right)^{-2 / 3}\left(\frac{v}{300 \mathrm{~km} / \mathrm{s}}\right)^{-2 / 3} \gamma^{-2 / 3} \\
& \times\left(\frac{B_{0}}{1 \mu \mathrm{G}}\right)^{2 / 3}\left(\frac{L_{\mathrm{max}}}{50 \mathrm{pc}}\right)^{-1 / 3}\left(\frac{\eta_{B}}{0.05}\right)^{1 / 2}\left(\frac{v_{A}}{30 \mathrm{~km} / \mathrm{s}}\right), \\
\Gamma_{\mathrm{diff}}= & \frac{2 \times 10^{-12}}{\mathrm{yr}}\left(\frac{m / q}{10^{6} \mathrm{GeV}}\right)^{1 / 3}\left(\frac{v}{300 \mathrm{~km} / \mathrm{s}}\right)^{4 / 3} \gamma^{-2 / 3}\left(\frac{B_{0}}{1 \mu \mathrm{G}}\right)^{-1 / 3}\left(\frac{H}{3 \mathrm{kpc}}\right)^{-2}, \\
\Gamma_{\mathrm{adv}}= & \frac{1 \times 10^{-8}}{\mathrm{yr}}\left(\frac{v_{\mathrm{adv}}}{30 \mathrm{~km} / \mathrm{s}}\right)\left(\frac{H}{3 \mathrm{kpc}}\right)^{-2} .
\end{aligned}
$$

Since charged DM is non-relativistic, advection dominates over diffusion except for the largest $m / q$ values considered in Fig. 1. However, all three rates are well below the growth rate in the range where the resonance condition is satisfied.

\subsection{Thermalisation rate}

The thermalisation time scale for a particle with charge $q_{1} e$, mass $m_{1}$ and velocity $v$ passing through a medium consisting of particles with charge $q_{2} e$, mass $m_{2}$ and density $n$ is given by

$$
t_{c}=\frac{m_{1} m_{2} v^{3}}{8 \pi q_{1}^{2} q_{2}^{2} e^{4} n_{e} \ln \Lambda},
$$

where we use $\ln \Lambda \sim 20$ as Gaunt factor [35]. The thermalisation is dominated by the warm ionized medium which has fractional volume $f_{\mathrm{WIM}}=0.15$, electron density $n_{e}=0.2 \mathrm{~cm}^{-3}$ and temperature $8 \times 10^{3} \mathrm{~K}$ [36]. Since the velocity of the charged DM, $v_{\text {vir }} \sim 300 \mathrm{~km} / \mathrm{s}$, is smaller than the electron velocity in the warm ionized medium, $v_{e} \sim 600 \mathrm{~km} / \mathrm{s}$, the charged DM will thermalise at a rate

$$
\Gamma_{\text {therm }}=\frac{1}{t_{c}} \sim \frac{7 \times 10^{-8}}{\mathrm{yr}}\left(\frac{m / q^{2}}{10^{6} \mathrm{GeV}}\right)^{-1}\left(\frac{v_{e}}{600 \mathrm{~km} / \mathrm{s}}\right)^{-3}\left(\frac{n_{e}}{0.2 \mathrm{~cm}^{3}}\right)\left(\frac{f_{\mathrm{WIM}}}{0.15}\right)
$$

in the thin disk. As visible from Fig. 1, this rate is much smaller than the other relevant rates. In addition, thermalisation only occurs in the thin Galactic disk, but the charged DM spend most of its time outside this region. Thus, thermalisation can be neglected in the present work.

\subsection{Supernova shock encounter rate}

The effect of supernova remnants on charged DM was studied in Ref. [22]. The expected rate at which charged DM particles in the Galactic disc will encounter supernova shocks is [22]

$$
\Gamma_{\mathrm{SH}}=\frac{4 \times 10^{-8}}{\mathrm{yr}}\left(\frac{R_{\mathrm{max}}}{40 \mathrm{pc}}\right)^{3}\left(\frac{R_{\mathrm{disc}}}{15 \mathrm{kpc}}\right)^{-2}\left(\frac{H_{\mathrm{disc}}}{300 \mathrm{pc}}\right)^{-1}\left(\frac{\Gamma_{\mathrm{SN}}}{0.03 \mathrm{yr}^{-1}}\right),
$$

This rate is much smaller than the growth rate for $m / q \lesssim 10^{15} \mathrm{GeV}$. Thus, the acceleration of charged DM by supernova shocks can be neglected.

\subsection{Injection rate of turbulence}

The turbulence is injected at scales $L_{\max } \sim 50-100 \mathrm{pc}$ through the source term $q_{W} \propto \delta\left(k-2 \pi / L_{\max }\right)$. Without the presence of charged DM, a Kolmogorov spectrum will develop. Thus, the source term can be found as

$$
q_{W}(k)=\frac{5 \times 10^{-11}}{\mathrm{yr}}\left(\frac{\eta_{B}}{0.05}\right)^{3 / 2}\left(\frac{v_{A}}{30 \mathrm{~km} / \mathrm{s}}\right)\left(\frac{L_{\max }}{50 \mathrm{pc}}\right)^{-1} \delta\left(k-2 \pi / L_{\max }\right) .
$$


Meanwhile, the rate for the absorption of wave power by charged DM can be estimated as

$$
\begin{aligned}
\int_{k_{\min }}^{k_{\max }} \mathrm{d} k & \Gamma_{\text {growth }} W \simeq-\int_{\gamma m v_{A}}^{\infty} \mathrm{d} p \frac{q e B_{0}}{p^{2} c} \Gamma_{\text {growth }} W \\
& =\frac{2 \times 10^{-4}}{\mathrm{yr}}\left(\frac{m / q}{10^{6} \mathrm{GeV}}\right)^{-1}\left(\frac{v_{\mathrm{vir}}}{300 \mathrm{~km} / \mathrm{s}}\right)^{2 / 3} \gamma^{2 / 3}\left(\frac{\eta_{B}}{0.05}\right)\left(\frac{L_{\max }}{50 \mathrm{pc}}\right)^{-2 / 3}\left(\frac{v_{A}}{30 \mathrm{~km} / \mathrm{s}}\right)^{2} .
\end{aligned}
$$

In the second step, we extended the integration region from the resonance up to infinity, which is admissible since the gradients of $f$ are strongly peaked. Moreover, we used $m v_{A} / p_{\text {vir }} \sim 0.1$ to obtain a numerical value of the resulting Gamma function. According to this simple estimate, the absorption rate of turbulence is larger than the injection rate for $m / q \lesssim 10^{12} \mathrm{GeV}$, i.e. in all the range where the resonance condition is satisfied.

\section{Time evolution and observable consequences}

\subsection{Time evolution}

In order to better understand the process of cascading and absorption of wave power, we solve the timedependent diffusion equations (2.1) and (2.3) using the Crank-Nicholson scheme. As initial condition, we use the Maxwellian phase space distribution (3.3) with $n(z)=n_{0}$. From the discussions in the previous section, we know that the reacceleration is dominant, and we therefore neglect convective and spatial diffusive terms ${ }^{5}$ in the diffusion equation (2.1). Likewise, the growth due to the spatial gradient in Eq. (2.2) is subdominant and can be neglected.

For concreteness, we consider $m=10^{6} \mathrm{GeV}$ and $q=10^{-3}$ as an example. Moreover, we assume first that the charged DM abundance $\Omega_{\mathrm{cDM}}$ equals the total DM abundance $\Omega_{\mathrm{DM}}$, i.e. $f_{\mathrm{cDM}}=\Omega_{\mathrm{cDM}} / \Omega_{\mathrm{DM}}=1$. In the first row of figure 2 , we show how the wave power cascades to larger wave-numbers and how this affects the phase space density of the charged DM setting by hand the growth term $\Gamma_{\text {growth }}$ in Eq. (2.2) to zero: As the time increases, the turbulence injected around the scale $k_{\text {min }}$ cascades to larger and larger wave-numbers, forming a Kolmogorov power-law spectrum, while the phase-space density of charged DM develops an increasing high-energy tail. Switching on the growth term, however, the turbulent cascade stops at $k \sim q e B_{0} /\left(p_{\text {vir }} \times\right.$ few $)$, as shown in the second row. Note that in this case the momentum distribution of charged DM does not change drastically.

For a fully developed Kolmogorov spectrum we know from Eqs. (4.9) and (4.10) that all wave power is absorbed by the charged DM for $f_{\mathrm{cDM}}^{-1} m / q \lesssim 10^{12} \mathrm{GeV}$. For $m=10^{6} \mathrm{GeV}$ and $q=10^{-3}$ this transition occurs at $f_{\mathrm{cDM}} \sim 5 \times 10^{-4}$. To visualise the effect of partial absorption, we show in the third row of Fig. 2 the solution with $f_{\mathrm{cDM}}=10^{-5}$. The wave-spectrum $W(k)$ has now a drop at the resonance momentum, but recovers at larger $k$. This drop leads via Eq. (2.10) to a corresponding jump in the diffusion coefficient $D(p)$.

\subsection{Observable consequences}

Turbulence injected at large scales $L_{\max } \sim 50-100 \mathrm{pc}$ cascades to smaller wave lengths creating in the inertial range a power-law spectrum. In the presence of charged DM, however, the wave power will be absorbed when the cascade reaches $k_{\mathrm{vir}} \sim q e B_{0} / p_{\mathrm{vir}}$. Since the growth rate is for $f_{\mathrm{cDM}}=1$ much larger than the damping rate, the entire wave energy will be absorbed such that no waves can cascade above $\sim k_{\text {vir }}$. Thus, for charged DM with $m / q \lesssim 10^{11} \mathrm{GeV}$ the cascading will stop at $k_{\text {vir }}$. Therefore no charged particles with momenta above $p_{\text {vir }}$ be able to resonate with Alfvén waves. Effectively, this would lead to a sudden drop in the diffusion coefficient which is not observed in the cosmic ray spectra [37]. Similarly, cosmic rays with momenta above $p_{\text {vir }}$ would not be isotropised by the GMF, in contradiction to the very low level of anisotropy observed [37]. Using the absence of anisotropies and a sudden drop in spectra observed above $\sim 0.1 \mathrm{GeV}$, one can exclude $10^{3} \mathrm{GeV} \lesssim m / q \lesssim 10^{11} \mathrm{GeV}$. Additionally, charged DM is subject to upper limits set by terrestrial direct detection experiments,

\footnotetext{
${ }^{5}$ These terms can in principle be taken into account by introducing a "leaky-box" loss term $f / T$ and source term $f_{\mathrm{vir}} / T$.
} 

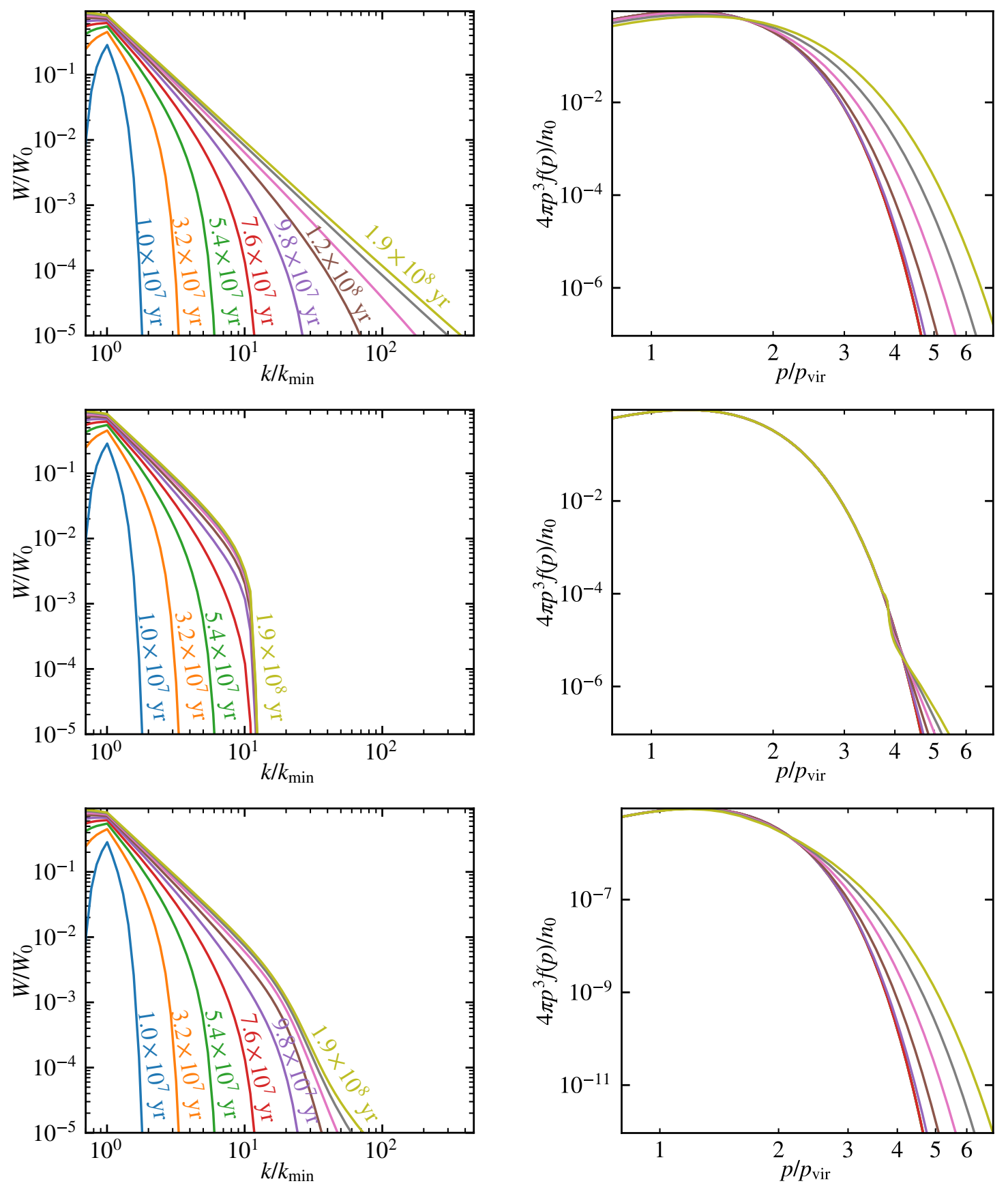

Figure 2. Time evolution of the wave power (left column) and the phase space density (right column). In the first row the growth rate is set to zero, while it is included in the second row. In the third row, the growth rate is included and $f_{\mathrm{cDM}}=10^{-5}$.

since in the absence of resonant Alfvén waves charged DM particles are exchanged freely between the Galactic disk and halo.

In Fig. 3, we show the upper limit in the $(m, q)$ parameter space for $f_{\mathrm{cDM}}=1$. The exclusion area for Xenon $1 \mathrm{~T}$ is taken from Ref. [38]. Furthermore, charged DM with $m / q^{2} \lesssim 10^{5} \mathrm{GeV}$ would have collapsed into the disc and is thus excluded [22]. Additional constraints are discussed in, e.g., 
Refs. [18, 22, 39].

Finally, we note that for $f_{\mathrm{CDM}}<1$ most existing exclusion limits become weaker. In the case of reacceleration, however, we found that the exclusion is limited by the resonance condition and holds as long as $f_{\text {cDM }}^{-1} m / q \lesssim 10^{12} \mathrm{GeV}$. Allowing for a partial transmission of wave-power to larger scales can further increase the excluded region in $f_{\mathrm{cDM}}$. For $f_{\mathrm{CDM}} \lesssim 10^{-6}$, the acceleration of charged DM will disturb its momentum distribution, and the methods to derive exclusion limits for charged cosmic rays worked out in Ref. [22] might be applied.

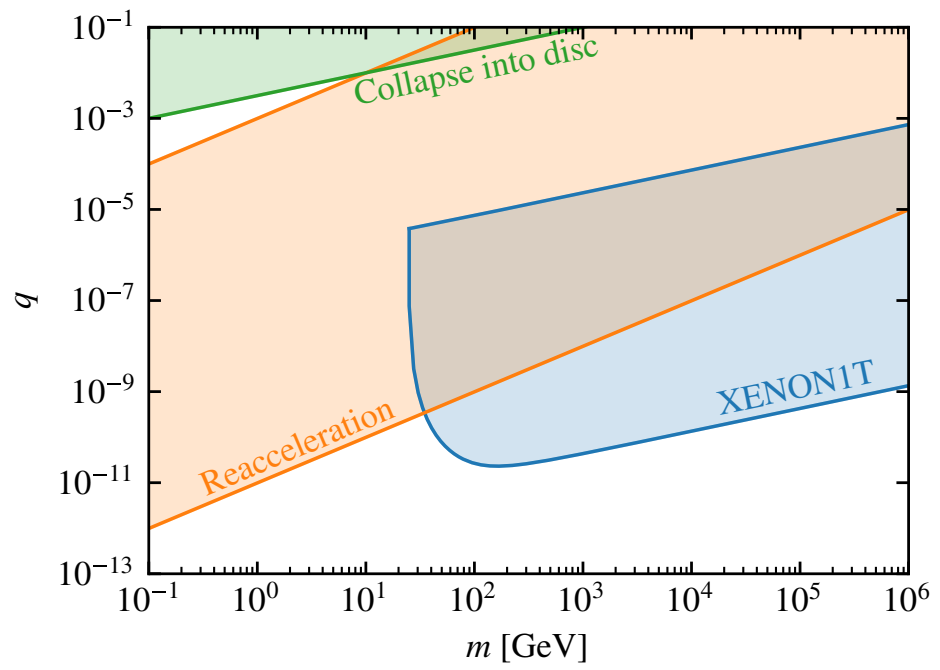

Figure 3. Exclusion plot of charged DM in the mass-charge plane for $f_{\mathrm{cDM}}=1$. The orange area is excluded due to the unobserved drastic change in the diffusion coefficient expected due to reacceleration of charged DM. The Xenon 1T 90\% exclusion (blue area) is obtained from Ref. [38], and the exclusion due to possible collapse into the disc is obtained from Ref. [22].

\section{Conclusions}

In this work we have shown that the feedback due to second-order Fermi acceleration is an important effect that must be taken into account when analysing the propagation of charged dark matter. The growth rate turns out to be dominant at mass to charge ratios $m / q \lesssim 10^{15} \mathrm{GeV}$. As such, the absorption of Alfvén waves will stop the cascading of wave-power to smaller scales around the Larmor radius of the charged particles, $k_{\text {res }} \sim 1 / R_{L}$. This will in turn imply a significant and sudden change in the diffusion coefficient for ordinary cosmic rays at this scale. This unobserved consequence leads to the excluded region $10^{3} \mathrm{GeV} \lesssim m / q \lesssim 10^{11} \mathrm{GeV}$. Even more, this limit remains fixed as long as the charged dark matter abundance $f_{X}=\Omega_{\mathrm{cDM}} / \Omega_{\mathrm{DM}}$ satisfies $f_{\mathrm{cDM}}^{-1} m / q \lesssim 10^{12} \mathrm{GeV}$.

\section{References}

[1] A. Yu. Ignatiev, V. A. Kuzmin and M. E. Shaposhnikov, Is the Electric Charge Conserved?, Phys. Lett. 84B (1979) 315.

[2] L. B. Okun, M. B. Voloshin and V. I. Zakharov, Electrical neutrality of atoms and Grand Unification models, Phys. Lett. 138B (1984) 115.

[3] H. Georgi, P. H. Ginsparg and S. Glashow, Photon Oscillations and the Cosmic Background Radiation, Nature 306 (1983) 765.

[4] B. Holdom, Two U(1)'s and Epsilon Charge Shifts, Phys. Lett. 166B (1986) 196. 
[5] M. I. Dobroliubov and A. Yu. Ignatiev, Millicharged particles, Phys. Rev. Lett. 65 (1990) 679.

[6] P. Fayet, Extra U(1)'s and New Forces, Nucl. Phys. B 347 (1990) 743.

[7] A. De Rujula, S. Glashow and U. Sarid, Charged dark matter, Nucl. Phys. B 333 (1990) 173.

[8] J. D. Bowman, A. E. E. Rogers, R. A. Monsalve, T. J. Mozdzen and N. Mahesh, An absorption profile centred at 78 megahertz in the sky-averaged spectrum, Nature 555 (2018) 67 [1810.05912].

[9] R. Barkana, Possible interaction between baryons and dark-matter particles revealed by the first stars, Nature 555 (2018) 71 [1803.06698].

[10] E. D. Kovetz, V. Poulin, V. Gluscevic, K. K. Boddy, R. Barkana and M. Kamionkowski, Tighter limits on dark matter explanations of the anomalous EDGES $21 \mathrm{~cm}$ signal, Phys. Rev. D 98 (2018) 103529 [1807.11482].

[11] J. B. Muñoz and A. Loeb, A small amount of mini-charged dark matter could cool the baryons in the early Universe, Nature 557 (2018) 684 [1802.10094].

[12] M. P. Hertzberg and M. Sandora, Dark Matter and Naturalness, JHEP 12 (2019) 037 [1908.09841].

[13] H. Liu, N. J. Outmezguine, D. Redigolo and T. Volansky, Reviving Millicharged Dark Matter for 21-cm Cosmology, Phys. Rev. D 100 (2019) 123011 [1908.06986].

[14] F. Kahlhoefer and E. Urdshals, On dark atoms, massive dark photons and millicharged sub-components, 2001.04492.

[15] M. Fabbrichesi, E. Gabrielli and G. Lanfranchi, The Dark Photon, 2005.01515.

[16] A. Filippi and M. De Napoli, Searching in the dark: the hunt for the dark photon, Rev. Phys. 5 (2020) $100042[2006.04640]$.

[17] S. Davidson, B. Campbell and D. C. Bailey, Limits on particles of small electric charge, Phys. Rev. D43 (1991) 2314.

[18] S. D. McDermott, H.-B. Yu and K. M. Zurek, Turning off the Lights: How Dark is Dark Matter?, Phys. Rev. D 83 (2011) 063509 [1011.2907].

[19] L. Chuzhoy and E. W. Kolb, Reopening the window on charged dark matter, JCAP 07 (2009) 014 [0809.0436].

[20] F. Sanchez-Salcedo, E. Martinez-Gomez and J. Magana, On the fraction of dark matter in charged massive particles (CHAMPs), JCAP 02 (2010) 031 [1002.3145].

[21] J.-T. Li and T. Lin, Dynamics of millicharged dark matter in supernova remnants, 2002.04625.

[22] D. Dunsky, L. J. Hall and K. Harigaya, CHAMP Cosmic Rays, JCAP 1907 (2019) 015 [1812.11116].

[23] V. L. Ginzburg and S. I. Syrovatskii, The origin of cosmic rays. New York: Gordon and Breach, 1969.

[24] J. Skilling, Cosmic ray streaming - I. Effect of Alfvén waves on particles., Mon. Not. Roy. Astron. Soc. $172(1975) 557$.

[25] J. Skilling, Cosmic ray streaming - III. Self-consistent solutions., Mon. Not. Roy. Astron. Soc. 173 (1975) 255.

[26] D. Melrose, The emission and absorption of waves by charged particles in magnetized plasmas, Astrophysics and Space Science 2 (1968) 171.

[27] J. A. Eilek, Particle reacceleration in radio galaxies., Astrophys. J. 230 (1979) 373.

[28] Y. Zhou and W. H. Matthaeus, Models of inertial range spectra of interplanetary magnetohydrodynamic turbulence, Journal of Geophysical Research: Space Physics 95 (1990) 14881.

[29] Ptuskin, V. S. and Zirakashvili, V. N., Limits on diffusive shock acceleration in supernova remnants in the presence of cosmic-ray streaming instability and wave dissipation, A\&A 403 (2003) 1.

[30] J. Armstrong, B. Rickett and S. Spangler, Electron density power spectrum in the local interstellar medium, Astrophys. J. 443 (1995) 209.

[31] A. Chepurnov and A. Lazarian, Extending Big Power Law in the Sky with Turbulence Spectra from WHAM data, Astrophys. J. 710 (2010) 853 [0905.4413]. 
[32] F. Boulanger et al., IMAGINE: A comprehensive view of the interstellar medium, Galactic magnetic fields and cosmic rays, JCAP 08 (2018) 049 [1805.02496].

[33] A. Schekochihin, S. C. Cowley, W. Dorland, G. Hammett, G. Howes, E. Quataert et al., Kinetic and fluid turbulent cascades in magnetized weakly collisional astrophysical plasmas, Astrophys. J. Suppl. 182 (2009) 310 [0704.0044].

[34] A. Thornbury and L. O. Drury, Power requirements for cosmic ray propagation models involving re-acceleration and a comment on second order Fermi acceleration theory, Mon. Not. Roy. Astron. Soc. 442 (2014) 3010 [1404.2104].

[35] M. S. Longair, High Energy Astrophysics. Cambridge: University Press, 2011.

[36] D. P. Cox, The Three-Phase Interstellar Medium Revisited, Ann. Rev. Astron. Astrophys. 43 (2005) 337.

[37] M. Kachelrieß and D. Semikoz, Cosmic Ray Models, Prog. Part. Nucl. Phys. 109 (2019) 103710 [1904.08160].

[38] G. Belanger, A. Mjallal and A. Pukhov, Recasting direct detection limits within micrOMEGAs and implication for non-standard Dark Matter scenarios, 2003.08621.

[39] A. Caputo, L. Sberna, M. Frias, D. Blas, P. Pani, L. Shao et al., Constraints on millicharged dark matter and axionlike particles from timing of radio waves, Phys. Rev. D 100 (2019) 063515 [1902.02695]. 